\title{
НАРУШЕНИЕ ЗАКОНА СОХРАНЕНИЯ ЭНЕРГИИ В РЕЛЯТИВИСТСКОЙ ТЕОРИИ ГРАВИТАЦИИ
}

\author{
(Представил Я. Эйнасто)
}

В рамках релятивистской теории гравитации Логунова определяется нарушение закона сохранения энергии в свободно падающих физических лабораторнях.

1. Введение. В общей теории относительности (ОТО) Эйнштейна нет вполне удовлетворительного определения локальной плотности энергии. Физически это является следствием принципа эквивалентности (свободно падающему наблюдателю гравитационное поле кажется отсутствующим); математической причиной этого является нетензорный характер связности. А. А. Логунов с сотрудниками $\left[{ }^{1,2}\right]$ развивает релятивистскую теорию гравитации (РТГ), исключая принцип эквивалентности, но сохраняя нетензорный характер связности. В данной работе докажем, что вследствие нетензорного характера связности истинного пространства (ИП) Минковского энергия Логунова $t^{\text {ๆ0 }}$ становится несохраняющейся в наших лабораториях величиной, если учитывать абсолютный * характер гравитационного поля, в котором Солнечная система свободно падает.

2. Законы сохранения и криволинейные координаты. Напомним, что имеется принципиальное различие между законом сохранения электрического заряда и законом сохранения энергии. Первому закону можно придать форму закона сохранения не только в прямоугольных, но и в криволинейных координатах, второму - нет. Первый закон переносится автоматически на случай искривленного пространства-времени (ПВ), второй - нет.

Остановимся на некоторых деталях закона сохранения энергии. Пусть в галилеевых координатах тензор энергин-импульса (ТЭИ) удовлетворяет закону сохранения энергии ** $T^{\mu v}{ }_{, v}=0$. В криволинейных координатах форма закона сохранения энергии нарушается

$$
\sqrt{-\gamma} T^{0 v}{ }_{\mid v}=\left(\sqrt{-\gamma} T^{0 v}\right)_{, v}+\sqrt{-\gamma} \Gamma_{\sigma v}^{0} T^{\sigma v}=0
$$

\footnotetext{
* Словом абсолютный мы характеризуем те тензорные в РТГ величины, которые в ОТО являются нетензорными.

** Греческие буквы - индексы принимают значения $0,1.2,3$, латинскне $-1,2,3$; по дважды встречающемуся индексу производится суммирование; $\gamma_{\mu v}-$ метрический тензор ИП Минковского, $g_{\mu v}$ - метрический тензор (эффективного) пространства Римана, $\Gamma_{\mu v}^{\alpha}(\gamma)$ и $\Gamma_{\mu v} \alpha(g)$ - соответствуюшие символы Кристоффеля; $\gamma=\operatorname{det} \gamma_{\mu v}$, $g=\operatorname{det} g_{\mu v}, T \alpha \beta, \beta-$ обычная пронзводная, $T \alpha \beta ; \beta-$ ковариантная производная в пространстве Римана, $\left.T \alpha \beta\right|_{\beta}-$ коварнантная производная в криволинейных координатах ИП Минковского. Пользуемся единицами, в которых скорость света $c=1$ и эйнштейновская гравитационная постоянная $x=8 \pi$.
} 


$$
\frac{d}{\mathrm{dt}} \int_{V} \sqrt{-\gamma} T^{00} d^{3} x=-\oint_{\partial V} \sqrt{-\gamma} T^{0 i} d \Sigma_{i}-\int_{V} \sqrt{-\gamma} \Gamma_{\sigma \nu}^{0} T^{\sigma v} d^{3} x,
$$

где $\partial V$ - граница трехмерной области $V$, а $d \Sigma_{i}-$ элемент границы. Изменение энергии в рассматриваемом объеме $V$ не равняется потоку энергии через границу $\partial V$ объема.

Покажем, что свойство (1) тензора второго ранга имеет следующие последствия: в ОТО нет универсальных тензорных законов сохранения, а в РТГ законы сохранения для наших лабораторий нарушаются.

В конечных областях искривленного ПВ невозможно ввести галилеевы координаты и член $\sqrt{-g} \Gamma_{\sigma v}^{v}(g) T^{\sigma v}$ пишется в ОТО в нетензорной форме $\left(\sqrt{-g} \tau^{0 \alpha}\right), \alpha$, где $\tau^{0 \alpha}$ интерпретируется (в квазигалилеевых координатах) как плотность энергии-импульса гравитационного поля. Имеется нетензорный закон сохранения $\left[\sqrt{-g}\left(T^{0 \alpha}+\tau^{0 \alpha}\right)\right]_{, \alpha}=0$. В случае гравитационного излучения из $\tau^{\mu \nu}$ можно получить настоящий ТЭИ путем усреднения по нескольким длинам волн $\left[{ }^{3}\right]$. В случае статических полей удовлетворительной процедуры «тензоризации» $\tau^{\mu \nu}$ нет. Поэтому локализовать удается только энергию излучения, но не энергию статического поля.

В теории гравитации Логунова галилеевы в окрестности Солнечной системы координаты являются криволинейными с точки зрения ИП Минковского координатами, что ведет к нарушению законов сохранения в наших лабораториях.

$$
\sqrt{-\gamma}^{0 v}{ }_{\mid v}=\left(\sqrt{-\gamma} t^{0 v}\right)_{, v}+\sqrt{-\gamma} \Gamma_{\alpha v}^{0}(\gamma) t^{\alpha v}=0
$$

где $t^{\mu \nu}$ - метрический тензор энергии-импульса ИП Минковского. *** Вычислим $\Gamma_{\alpha \nu}^{0}(\gamma)$ в инерциальных с точки зрения ОТО системах отсчета.

\section{3. Галилеевы координаты ОТО как криволинейные координаты РТГ.} Предположим, что наша лаборатория свободно падает в слабом однородном гравитационном поле, ньютоновский потенциал которого $U=$ $=-G \bar{x}$. Соответствующий приближенный линейный элемент в гармонических координатах следующий $\left(\left[{ }^{4}\right], \S 55\right)$ :

$$
d s^{2}=(1+2 G \bar{x}) d \bar{t}^{2}-(1-2 G \bar{x})\left(d \bar{x}^{2}+d \bar{y}^{2}+d \bar{z}^{2}\right) .
$$

Этот же линейный элемент в рассматриваемом нами приближении можно получить из метрики Минковского

$$
d s^{2}=c^{2} \mathrm{dt}^{2}-d x^{2}-d y^{2}-d z^{2}
$$

с помощью преобразований координат

$$
\begin{aligned}
x & =\bar{x}+\frac{1}{2} G \bar{t}^{2}-\frac{1}{2} G\left(\bar{x}^{2}-\bar{y}^{2}-\bar{z}^{2}\right)+O\left(G^{2}\right), \\
y & =\bar{y}-G \bar{x} \bar{y}+O\left(G^{2}\right), \\
z & =\bar{z}-G \bar{x} \bar{z}+O\left(G^{2}\right), \\
t & =\bar{t}+G \bar{x} \bar{t}+O\left(G^{2}\right) .
\end{aligned}
$$

\footnotetext{
*** В РТГ можно получить законы сохранения энергии, переходя от тензорных величин $t \alpha \beta$ к векторным $t \alpha{ }_{\beta} \xi^{\beta}$, где $\xi^{\beta}$ вектор Киллинга. Но вектор $\xi^{\beta}$ описывает ускоренную относительно Солнечной системы (точнее, инерциальную относительно ИП Минковского) лабораторию и $t^{0}{ }_{\beta} \xi^{\beta}$ не является энергией в реальных, падающих свободно во внешних гравитационных полях лабораториях. Так как нас интересуют свободно падающие лаборатории, то вектор $t \alpha_{\beta} \xi$ далее рассматривать не будем. Отметим только, что в случае эффективной метрики (3) имеем $\bar{\gamma}_{\alpha \beta}=\operatorname{diag}(1,-1,-1,-1), \bar{\xi} \alpha=$ $=(1,0,0,0)$, а в интересующих нас системах отсчета, заданных координатами (5) $x^{\alpha}=x^{\alpha}(\bar{x} \beta)$, имеем $\xi \alpha=(1+G x,+G t, 0,0)$.
} 
Символ $O\left(G^{2}\right)$ обозначает члены, пропорциональные $G^{2}$. Иногда вместо этого символа ставим три точки или просто опускаем его: мы ограничиваемся всегда наименьшим неисчезающим приближением.

Принцип эквивалентности ОТО позволяет рассматривать систему отсчета (4) как инерциальную и пользоваться в ней всеми законами специальной теории относительности (СТО), включая законы сохранения энергии. В РТГ система отсчета (4) неинерциальная, а используемые в ней координаты $x^{\alpha}$ нелинейные функции (5) галилеевых координат $\bar{x}^{\beta}$ ИП Минковского. Пользуясь законами преобразования символов Кристоффеля, получаем

$$
\begin{aligned}
& \Gamma_{\alpha \beta}^{\mu}(\gamma)=-\frac{\partial^{2} x^{\mu}}{\partial \bar{x}^{\alpha} \partial \bar{x}^{\beta}}+O\left(G^{2}\right), \\
& \Gamma_{\alpha \beta}^{0}(\gamma)=-G\left(\delta_{\alpha 1} \delta_{\beta 0}+\delta_{\alpha 0} \delta_{\beta 1}\right)+O\left(G^{2}\right) .
\end{aligned}
$$

Метрику $\gamma_{\mu v}$ можно вычислить и непосредственно. Определяя из (5) обратные функции $\bar{x}^{\alpha}=\bar{x}^{\alpha}\left(x^{\beta}\right)$ и подставляя их в (3), имеем

$$
g_{00}=(1+2 G x-2 G x+\ldots), \quad g_{i k}=(-1+2 G x-2 G x) \delta_{i k}+\ldots .
$$

В ОТО на основе принципа эквивалентности члены $2 G x$ и $-2 G x$ сокращаются. В РТГ такая операция недопустима. Член $2 G x$ определяет гравитационное поле типа Максвелла-Фарадея, а член - $G x$ относится к метрическому тензору ИП Минковского:

$$
\gamma_{00}=(1-2 G x), \quad \gamma_{i k}=(-1-2 G x) \delta_{i k} .
$$

4. Тензоры энергии-импульса. Напомним определения ТЭИ. Законам сохранения энергии придают в РТГ тензорную форму, абсолютизируя разложение

$$
\sqrt{-g} g^{\mu v}=\sqrt{-\gamma} \gamma^{\mu v}+\sqrt{-\gamma} \Phi^{\mu v}
$$

где $\Phi^{\mu v}$ - тензор гравитационного поля относительно ИП Минковского. Плотность функции Лагранжа следующая [ $\left.{ }^{1}\right]$ :

$$
L=L_{g}\left(\sqrt{-\gamma} \gamma^{\mu v}, \sqrt{-\gamma} \Phi^{\mu v}\right)+L_{M}\left(\sqrt{-g} g^{\mu v}, \Phi_{A}\right)
$$

Здесь $L_{g}$ - плотность функции Лагранжа гравитационного поля, $L_{M}-$ плотность функции Лагранжа вещества, $\Phi_{A}-$ поля вещества. Под веществом мы понимаем все виды материи, за исключением гравитационной. ТЭИ гравитационного поля определяется следующим образом: $\sqrt{-\gamma} t_{g}^{\mu \nu}=-2 \delta L_{g} / \delta \gamma_{\mu v}$, он является тензором ИП Минковского и мерой его превращений может быть только тензор этого же пространства. Поэтому определяется $\sqrt{-\gamma} t_{M}^{\mu \nu}=-2 \delta L_{M} / \delta \gamma_{\mu \nu}$, хотя на основе принципа геометризации РТГ движение всех видов вещества можно описать в эффективном римановом пространстве, не прибегая к разложению (8). Выполняется закон (не) сохранения

$$
t^{\mu v}{ }_{l v}=0, \quad t^{\mu v}=t_{g}^{\mu v}+t_{M}^{\mu v} .
$$

Наряду с $t^{\mu \nu}$ можно определить обычный метрический ТЭИ (эффективного) **** пространства Римана $\sqrt{-g} T^{\mu v}=-2 \delta L_{M} / \delta g_{\mu v}, T^{\mu v}{ }_{; v}=0$.

**** Слово эффективный заключено в скобки, когда сқазанное относится как к QTO, тақ и к РТГ. 
5. Предсказываемое теорией Логунова нарушение закона сохранения энергии. Рассмотрим случай, когда $t_{g \mid v}^{0 v}=0$. Из выражений (2) и (6) следует, что нарушение закона сохранения энергии в наших лабораториях имеет следующую форму:

$$
\left(\sqrt{-\gamma} t_{M}^{0 \alpha}\right)_{, \alpha}=2 \sqrt{-\gamma} G t_{M}^{01}
$$

Здесь $G$ - абсолютное ускорение Солнечной системы в локально однородном фоновом гравитационном поле $U=-G \bar{x}$. Это поле порождено Галактикой и другими неоднородностями в распределении масс, обнаруженных вплоть до расстояний 300 Мпс.

В более или менее чистом виде система отсчета (4) реализуется в свободно гравитирующей, невращающейся космической лаборатории, если вычесть эффекты, связанные с вращением и гравитационным полем Земли. Нужно учесть также то обстоятельство, что кроме свободного падения в фоновом поле $U=-G \bar{x}$, Земля падает свободно еще в гравитационном поле Солнца с ускорением $0,6 \mathrm{~cm} / \mathrm{c}^{2}$. Направление последнего ускорения, в отличие от $G$, периодически меняется.

6. Лифт Эйнштейна. Будем называть падающую в однородном гравитационном поле лабораторию лифтом Эйнштейна. В лифте (эффективное) пространство Римана плоское, в нем были введены галилеевы координаты (4), $g_{\mu \nu}=\operatorname{diag}(1,-1,-1,-1)$, в то время как метрика ИП Минковского $\gamma_{\mu \nu}$ дана выражениями (7). Функция Лагранжа вещества $L_{M}=L_{M}\left(\sqrt{-g} g^{\mu v}, \Phi_{A}\right)$ переходит в лифте в функцию Лагранжа СТО, метрический ТЭИ эффективного риманова пространства $T^{\mu v}$ - в метрический ТЭИ СТО, удовлетворяющий закону сохранения энергии.

7. Движение вещества в лифте Эйнштейна с точки зрения РТГ. Рассмотрим вопрос, можно ли по нарушению закона сохранения энергии (9) определить ускорение G лифта. А. А. Логунов ([ $\left.{ }^{5}\right]$, с. 7$)$ пишет: «Согласно принципу геометризации, движение вещества под действием гравитационного поля $\Phi^{\mu v}$ в пространстве Минковского с метрикой $\gamma^{\mu \nu}$ тождественно его движению в эффективном римановом пространстве с метрикой $g^{\mu \nu}$. Метрический тензор $\gamma^{\mu v}$ пространства Минковского и тензор гравитационного поля $\Phi^{\mu \nu}$ являются первичными понятиями, а риманово пространство и его метрика $g^{\mu v}-$ вторичными ...». В рассматриваемом нами случае лифта Эйнштейна эффективное пространство Римана - это плоское ПВ (4). Из вышеприведенной цитаты следует, что движение вещества в любом однородном гравитационном поле тождественно его движению в лифте Эйнштейна. Далее А. А. Логунов ([ $\left.{ }^{6}\right]$, с. 14$)$ пишет: «B нашей теории (РТГ) физическая геометрия определяется не на основе изучения движения света и пробных тел, а на основе общих динамических свойств материи - еe законов сохранения, которые не только имеют фундаментальное значение, но и экспериментально проверяемы». Исходя из этих соображений определяются ТЭИ ИП Минковского $t_{M^{\mu \nu}}$ и $t_{g}{ }^{\mu v}$. Неинерциальное с точки зрения РТГ движение лифта Эйнштейна сказывается в нарушении закона сохранения энергии (9). Но каким образом определить ускорение лифта, когда движение любого вида вещества не зависит от $G$ и удовлетворяет известным в СТО законам сохранения $T^{\mu v}{ }_{, v}=0$ ? На наш взгляд, на этот вопрос имеется только один ответ: экспериментально измеряемым (наблюдаемым) является ТЭИ СТО $T^{\mu \nu}$, частный вид ТЭИ эффективного риманова пространства, а не $t_{M}^{\mu \nu}$.

Переходим к энергии гравитационного поля $t_{g}^{\mu v}$. Энергию гравитационного поля можно измерить, превратив ее в другие виды энергии. 
Но так как мерой превращенной энергии является ненаблюдаемая энергия $t_{M}^{00}$, то ненаблюдаемой является и сама энергия гравитационного поля.

\section{8. Непревращаемость энергии постоянного гравитационного поля.} В случае эффективной метрики (4) имеем

$$
t_{g}^{00}=\frac{7}{32 \pi^{2}} G^{2}+O\left(G^{3}\right) .
$$

Эта энергия не может превращаться в энергию вещества $T^{00}$, так как из уравнений Эйнштейна следует, что в плоском ПВ $T^{\mu v}=0$.

9. Вывод. В РТГ ИП Минковского не является экспериментально наблюдаемым и, по-видимому, РТГ не предлагает окончательного решения проблемы энергии гравитационного поля.

\section{Л ИТ Р Р А У Р А}

1. Логунов А. А., Мествиришвили М. А. // ТМФ, 1984, 61, 327-346.

2. Logunov, A. A., Mestvirishvili, M. A. // Prog. Theor. Phys., 1985, 74, 31-50.

3. Isaacson, R. A. Gravitational Radiation in the Limit of High Frequency. University of Maryland, 1967 (Technical report № 657, Ch. III), 44-67.

4. Фок В. А. Теория пространства, времени и тяготения. М., ГИТТЛ, 1955.

5. Логунов A. A. Новые представления о пространстве-времени и гравитации. М., МГУ, 1986.

6. Логунов А. А. Релятивистская теория гравитации и новые представления о пространстве-времени. М., МГУ, 1986.

Институт астрофизики и физики атмосферы Академии наук Эстонской ССР

\section{Поступила в редакцию} $29 / 1 \quad 1988$

V. UNT

\section{ENERGIA JAAVUSE SEADUSTE RIKKUMINE RELATIVISTLIKUS GRAVITATSIOONITEOORIAS}

On näidatud, et erinevalt üldrelatiivsusteooriast ennustab Logunovi relativistlik gravitatsiooniteooria, et vabalt langevates laboratooriumides energia jäävuse seadused ei kehti.

V. UNT

\section{VIOLATION OF CONSERVATION LAW OF ENERGY IN RELATIVISTIC THEORY OF GRAVITATION}

Within the framework of the Logunov's relativistic theory of gravitation the violation of the conservation law of energy is found in freely falling frames of references, 